EPJ manuscript No.

(will be inserted by the editor)

\title{
From Regge Behavior to DGLAP Evolution
}

\author{
L. Csernai ${ }^{1}$, L. Jenkovszky ${ }^{2}$, K. Kontros ${ }^{3}$, A. Lengyel ${ }^{3}$, V. Magas ${ }^{4}$, and F. Paccanoni ${ }^{5}$
}

1 Section for Theoretical Physics, Department of Physics, University of Bergen, Allegaten 55, 5007 Bergen, Norway (e-mail: csernai@fi.uib.no)

2 Bogolyubov Institute for Theoretical Physics, National Academy of Sciences of Ukraine, 01143 Kiev, Ukraine (e-mail: jenk@gluk.org)

3 Institute of Electron Physics, National Academy of Sciences of Ukraine, Universitetska 21, 88016 Uzhgorod, Ukraine (e-mail: jeno@kontr.uzhgorod.ua, sasha@len.uzhgorod.ua)

4 Center for Physics of Fundamental Interactions, Instituto Superior Tecnico, Av. Rovisco Pais, 1049-001 Lisbon, Portugal (e-mail: vladimir@cfif.ist.utl.pt)

5 Dipartimento di Fisica, Università di Padova, Instituto Nazionale di Fisica Nucleare, Sezione di Padova via F. Marzolo 8, I-35131 Padova, Italy (e-mail: paccanoni@pd.infn.it)

Received: date / Revised version: date

\begin{abstract}
We study the interface between Regge behavior and DGLAP evolution in a non-perturbative model for the nucleon structure function based on a multipole pomeron exchange. This model provides the input for a subsequent DGLAP evolution that we calculate numerically. The soft input and its evolution give a good fit to the experimental data in the whole available range of $x$ and $Q^{2}$.
\end{abstract}

\section{Introduction}

There is a growing consensus on that nucleon structure functions (SF) at small virtualities $Q^{2}$ are Regge behaved and that at large $Q^{2}$ they follow QCD evolution [1]. The two regimes are incompatible because the DGLAP evolution [2], in general, changes the functional form of any Regge behavior (Regge models compatible with QCD evolution in a limited range of $Q^{2}$ will be mentioned below). What remains completely unclear is the border between the two.

Phenomenologically, the Regge pole approach to deep inelastic scattering implies that the structure functions are sums of powers in $x$, modulus logarithmic terms, each with a $Q^{2}$-dependent residue factor. The rapid rise in $Q^{2}$ of the structure functions, observed at HERA, initially was considered as a sign of departure from the standard Regge behavior. The reason was that the data, when fitted by a single "Regge-pomeron" term $\sim x^{-\lambda}$, where $\lambda$ is the pomeron intercept minus one, show that $\lambda=\frac{d}{d \ln 1 / x} \ln F_{2}\left(x, Q^{2}\right)$ definitely rises with $Q^{2}$. If so, Regge factorisation should be broken, since the "effective" pomeron trajectory appears to be $Q^{2}$-dependent.

The above categoric conclusion was however based on a parametrisation of the SF by a single pomeron term, e.g. $\sim x^{-\lambda}$. It is evident, that additional, decreasing ("subleading") terms, typical of any Regge pole model, will modify the properties of the leading one (pomeron). Moreover, by increasing the number of the free parameters and with complicated residue functions one may fit the data for arbitrarily large values of $Q^{2}$ in a factorised form, with- out introducing any $Q^{2}$-dependent trajectory, although it makes little sense to reach good $\chi^{2}$ at any rate with an indefinite number of free parameters introduced without any physical justification.

The (in)dependence of the Regge behavior (trajectory) from the external masses (virtualities) comes from the following arguments. The Regge asymptotic behavior

$$
A\left(s, t, Q^{2}\right)=\xi(t) \beta\left(t, Q^{2}\right)\left(\frac{s}{s_{0}}\right)^{\alpha(t)}
$$

where $\xi(t)$ is the signature factor, $\beta\left(t, Q^{2}\right)$ is the residue function, $\alpha(t)$ is the Regge trajectory and $s, t$ are the Mandelstam variables, comes from a Sommerfeld-Watson transform over the partial-wave amplitude (see for example [3])

$$
a\left(l, t, Q^{2}\right)=\frac{\beta\left(t, Q^{2}\right)}{l-\alpha(t)} .
$$

By Regge factorisation, $Q^{2}$-dependence can be introduced in the residue, but not in the trajectory.

In this paper we are mainly interested in the small$x$ or high-energy behavior of the structure functions and photon-proton total cross sections, therefore we concentrate on the pomeron (in terms of the Regge pole model) or singlet component of the SF, although, clearly, good fits and the evolution equation require the whole kinematical region in $x$ to be properly covered. Model for the pomeron, or singlet SF, and non leading contributions, or non-singlet SF, will be presented in Sec. 2. 
Even though the role of the QCD evolution was studied in a great number of papers (see e.g. [1] and references therein) the border and the interface between the non-perturbative Regge dynamics and perturbative QCD evolution is still determined merely by trial and error. Depending on the scope of the study, one may prefer either approximate solutions [5, 6, ,7 of the DGLAP equation [2] or computer calculations, for which purpose efficient numerical solutions and relevant computer codes are available 8 .

Explicit analytical solutions are attractive for their simplicity and physical transparency. In paper [6], for example, the transition due to the evolution from a logarithmic rise in $x$ (dipole model) to a power-like was shown explicitly by means of an approximate solution of the DGLAP equation. Another example, in the Regge framework, is given by the CKMT model [9,10].

Of particular interest are self-consistent solutions of the DGLAP evolution equations [5], i.e. those whose functional form does not change under the evolution. The product of logarithms (in $x$ and in $Q^{2}$ ) proved to be particularly stable. In ref. [7] this stability for the calculated $Q^{2}$-dependent coefficients, appearing in front of the factors logarithmic in $x$, was checked experimentally. The stability of the combination of the logarithms

$$
F_{2}\left(x, Q^{2}\right)=a+m \xi, \quad \xi=\ln \frac{Q^{2}}{Q_{0}^{2}} \ln \frac{x_{0}}{x}
$$

was noticed also by Buchmüller and Haidt 11] from phenomenological analyses of the data. Ref. Hit approaches this problem in a different spirit. The inclusion of a "hardpomeron" term, with Regge intercept about 1.4, allows for a fit to structure functions with a $\chi^{2}$ per data point near 1 . Moreover this new term is self-consistent, in the sense that a phenomenological parametrization of its $Q^{2}$ dependence, at small $Q^{2}$, turns out to be in agreement with perturbative evolution. However, the resulting dynamics here is different from the one necessary to describe hadron-hadron scattering.

Aiming at an unbiased extraction of the $Q^{2}$-dependent factors appearing in the structure functions, the authors in 12] have fitted them for a number of different models of the pomeron, each appended by subleading terms as well as a large- $x$ factor. The numerical values of the fitted coefficients for a large number of fixed values of $Q^{2}$ (Figs. 1, 2, 3 in [12], see also Fig. 1 in ref. 13) can be used as "experimental values", independent of any evolution scheme.

The aim of this paper is to check the onset of QCD evolution for the model of small- $x$ SF that was discussed earlier in [7, 12]. This model contains a small (minimal in our opinion) number of free parameters, still it is feasible and, supplemented by an evolution scheme, may have numerous applications.

Our approach concerns the class of phenomenological parametrisation of structure functions describing the experimental data in the non-perturbative region; using this parametrisation as input to the DGLAP equations one obtains a description of the experiment in the whole kine- matic range [9,4]. This genuine evolution, in contrast to global fits [14] 15], does not use data at large $Q^{2}$ to constrain the fit already at small $Q^{2}$. In comparison to the DL [4] model, where the pomeron has a hard nature, we choose a multipole pomeron with unit intercept.

In the present paper the evolution is calculated numerically by means of the codes developed and published by Miyama and Kumano [8].

\section{Multipole pomeron model}

We analyze a unit intercept Multipole pomeron, rather than a supercritical one as in the CKMT case [9]. Here Multipole pomeron (MP) means that the pomeron is a multipole instead of just a simple pole. The advantages of this approach, both for hadron-hadron and for leptonhadron scattering, were tested and discussed in numerous papers on that subject (see [1, 7, 12, 13, 16, 17, 18, 19] and references therein). The main point is that rising cross sections (and rising SF) can be produced with a unit pomeron intercept. The number and the relative weight of the contributing multipoles is a very important question. In QCD it was studied in ref. 20]. The conclusion of both the theory [20] and phenomenology is that a limited (moreover, small!) number of multipoles is sufficient in the present energy (or $x, Q^{2}$ ) range. In fact, a dipole (logarithmic rise), eventually with a minor tripole contribution (squared logarithm) accounts for the large part of the data (higher order poles do not manifest).

The dipole (and tripole) pomeron are typically "soft" objects. For the total cross sections they give fits close to those of a "soft" supercritical, i.e. with $\alpha(0) \approx 1.08$, pomeron. However, with an extra variable $-t$ in hadronhadron processes or $Q^{2}$ in lepton-hadron reactions - the differences become essential. The result of the analysis below, including QCD evolution, makes this comparison more complete.

Both non-perturbative inputs are written as sums of singlet and non-singlet terms:

$$
F_{2}\left(x, Q^{2}\right)=F_{S}\left(x, Q^{2}\right)+F_{N S}\left(x, Q^{2}\right) .
$$

We use the same non-singlet term taking it from ref. [10] as

$$
\begin{gathered}
F_{N S}\left(x, Q^{2}\right)=D \cdot x^{1-\alpha_{R}} \cdot(1-x)^{n\left(Q^{2}\right)} \cdot\left(\frac{Q^{2}}{Q^{2}+b}\right)^{\alpha_{R}}, \\
n\left(Q^{2}\right)=\frac{3}{2} \cdot\left(1+\frac{Q^{2}}{Q^{2}+c}\right)
\end{gathered}
$$

where, contrary to the original paper [10], we disregard the difference between the light quarks.

The singlet component of the SF, corresponding to a multipole (single+double+triple) pomeron is a sum of logarithms: 
Table 1. Parameters of the MP model

\begin{tabular}{cc}
\hline MP model & values of the parameters \\
\hline$A$ & 0.8286 \\
$B$ & $-0.1602 .10^{-1}$ \\
$C$ & $0.8208 .10^{-2}$ \\
$a$ & 0.7951 \\
$b$ & 1.263 \\
$c$ & 4.430 \\
$\alpha$ & 2.0 (fixed) \\
$\beta$ & 2.0 (fixed) \\
$\gamma$ & 0.7711 \\
$\alpha_{R}$ & 0.415 (fixed) \\
$D$ & 1.434 \\
$\chi^{2} / d o f$ & 1.08 \\
\hline
\end{tabular}

$$
\begin{gathered}
F_{S}\left(x, Q^{2}\right)=Q^{2}\left[A\left(\frac{a}{a+Q^{2}}\right)^{\alpha}+B\left(\frac{a}{a+Q^{2}}\right)^{\beta} \log \left(\frac{Q^{2}}{x}\right)+\right. \\
\left.C\left(\frac{a}{a+Q^{2}}\right)^{\gamma} \log ^{2}\left(\frac{Q^{2}}{x}\right)\right](1-x)^{n\left(Q^{2}\right)+4}
\end{gathered}
$$

The same type of singlet component was used in 13 .

The real photon-proton total cross-section has the following form:

$$
\begin{gathered}
\sigma_{\gamma p}^{t o t}\left(W^{2}\right)=4 \pi^{2} \alpha_{E M} \cdot\left(A+B \cdot \log \left(W_{1}^{2}\right)+\right. \\
\left.C \cdot \log ^{2}\left(W_{1}^{2}\right)+D \cdot d^{-\alpha_{R}} \cdot\left(W_{1}^{2}\right)^{\alpha_{R}-1}\right)
\end{gathered}
$$

where $W_{1}^{2}=W^{2}-m_{p}^{2}$.

The parameters of (4)-(8) were determined from a combined fit of $\sigma_{\gamma p}^{t o t}$ for $W>4 \mathrm{GeV}$ and structure function in the range of $0.045 \mathrm{GeV}^{2} \leq Q^{2} \leq 5 \mathrm{GeV}$.

We performed these fits to the set of the experimental data [21 using eqs. (4)-(8). The values of the fitted parameters and of $\chi^{2}$ are quoted in Table 1.

We have a rather small (eight) number of free parameters. In the MP model we fix the reggeon intercept as in the CKMT model. Additionally $\alpha$ and $\beta$ were fixed being not sensitive to the fit. The resulting fits are shown in Figs. 1 and 2 .

As already mentioned, we chose the starting point for the QCD evolution in such a way as to have best fits with the smallest number of the free parameters. By trial we found that the value $Q_{0}^{2}=6 \mathrm{GeV}^{2}$ is preferable. In the numerical calculation, we assume that the only difference between the sea-quark and gluon distributions is a factor $G(1-x)^{-2}$ in accordance with the dimensional counting rules. The value of the constant $G$ is 3.8 , the number of flavors is $4, \lambda=200 \mathrm{MeV}$. In contrast to [4], in our case the ratio of the gluon distribution to the singlet quark distribution is smaller by a factor of two.

Figs. 2 and 3 show the extrapolation of the $Q^{2}$-dependent "soft" Regge input as well as the results of our numerical calculations of the DGLAP equation by means of the NLO brute-force method, developed by Miyama and Kumano in ref. 8], thus extended to the high values of $Q^{2}$, measured at 21]. The input coincides with the structure

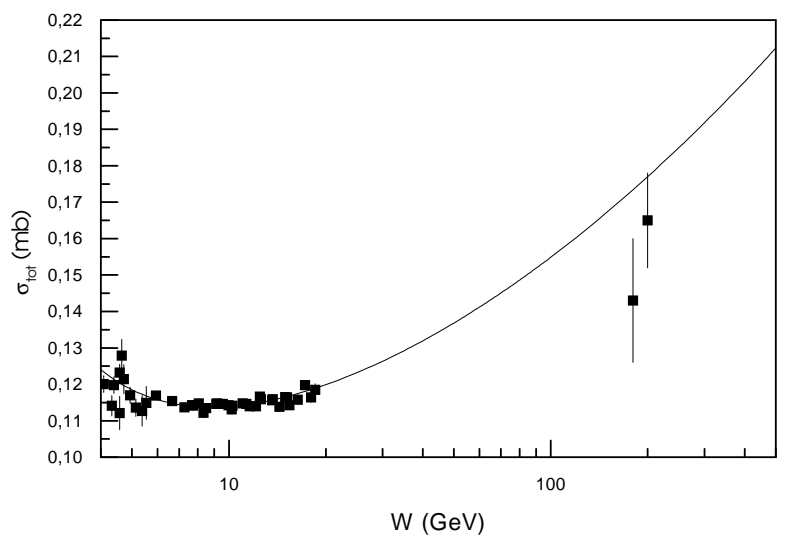

Fig. 1. Total $\gamma p$ cross-section versus $W$ in the MP model.

function itself, supplemented by the aforesaid gluon distribution, since the corrections implied by the $\overline{M S}$ scheme are small and we neglected them. Although we are primarily interested in the small- $x$ behavior, large- $x$ data, including those from fixed-target experiments [21] were also taken into account since they can influence the small$x$ behavior through integration in the evolution equation.

\section{Conclusions}

We show that the world's data on $F_{2}$, down to $Q^{2}=0$, are reasonably well described within the Regge model we have considered. The model succeed in reproducing the data for all $x$ and a large $Q^{2}$ interval, until $Q^{2} \approx 6 \mathrm{GeV}^{2}$, whereupon DGLAP equations must be used in order to describe correctly the $Q^{2}$ evolution. What happens at the border between Regge regime and QCD evolution is clarified in the last icon of Fig. 2 and the first three frames of Fig. 3. While the starting point for the DGLAP evolution has been chosen at $Q_{0}^{2}=6 \mathrm{GeV}^{2}$, the extrapolation of the "soft" Regge behavior gives a satisfactory fit of the data until $Q^{2} \approx 10 \mathrm{GeV}^{2}$. In Fig.3, one can see that the fit, based on eqs. (4)-(8), deteriorates when $Q^{2}$ increases and, at $Q^{2}=20 \mathrm{GeV}^{2}$, it is excluded by the data. There is however a region, a $Q^{2}$ interval, where the two regimes, Regge and perturbative QCD, are compatible. The comparison with other models, that consider only the small- $x$ region [4, 5, 6, [7, 11], shows that the region of compatibility shrinks when all the $x$-range is taken into account. To what extent this depends on the specific model we have chosen, is an interesting question and will be studied elsewhere.

To summarize, we find that the model described in eq. (7), for singlet SF, supplemented by a non-singlet component as in eq. (5), together with the DGLAP evolution, provides a simple and economic solution that could be useful for further practical applications, for example in nuclear physics. In addition, we can conclude that the soft input and its evolution give a good fit to the experimental data in whole available range of the variables $x$ and $Q^{2}$. 
L. Csernai et al.: From Regge Behavior to DGLAP Evolution

K. Kontros and A. Lengyel acknowledge the hospitality and support at the Bergen Computational Physics Laboratory (BCPL), where this work was started, while L. Jenkovszky thanks University and the INFN section in Padova, where it was completed.

\section{References}

1. M. Bertini et al., Rivista del Nuovo Cimento 19, (1996) 1.

2. V.N. Gribov, L.N. Lipatov, Sov. J. Nucl. Phys. 15, (1972) 438 and 675; G. Altarelli, G. Parisi, Nucl. Phys. B126, (1977) 298; Yu.L. Dokshitzer, Sov. Phys. JETP 46, (1977) 641.

3. P.D.B. Collins, Introduction to Regge Theory and High Energy Physics, Cambridge University Press (1977).

4. A. Donnachie, P.V. Landshoff, Phys. Lett. B437, (1998) 408; J.R. Cudell, A. Donnachie, P.V. Landshoff, Phys. Lett. B448, (1999) 281; A. Donnachie, P.V. Landshoff, Perturbative $Q C D$ and Regge theory: closing the circle, arXiv: hepph/0111427, 29 Nov. 2001.

5. R.D. Ball, S. Forte, Phys. Lett. B336, (1994) 77; F. Paccanoni Note on the DGLAP evolution equation, in Strong Interaction at Long Distances (Hadronic Press), (1995) 66.

6. L. Jenkovszky, A. Kotikov, F. Paccanoni, Phys. Lett. B314, (1993) 421.

7. L. Jenkovszky, A. Lengyel, F. Paccanoni, Nuovo Cim. A111, (1998) 551.

8. M. Miyama, S. Kumano, Computer Physics Communication 94, (1996) 185.

9. A. Capella, A.B. Kaidalov, C. Merino, J. Tran Thanh Van, Phys. Lett. B337, (1994) 358.

10. A.B. Kaidalov, C. Merino, D. Pertermann, Eur. Phys. J. C20, (2001) 301.

11. W. Buchmüller, D. Haidt, Double-logarithmic Scaling of the Structure Function $F_{2}$ at small $x$, DESY 96-061, May 1996; D. Haidt, Nucl. Phys. B96, (Proc. Suppl.) (2001) 166.

12. P. Desgrolard, L. Jenkovszky, A. Lengyel, F. Paccanoni, Phys. Lett. B459, (1999) 265

13. J.R. Cudell, G. Soyez, Phys. Lett. B516, (2001) 77.

14. A. Cooper-Sarkar, ZEUS NLOQCD fits, arXiv: hepph/0110386, 30 Oct. 2001.

15. A.D. Martin, R.G. Roberts, W.J. Stirling, R.S. Thorne, Eur. Phys. J. C18, (2000) 117.

16. L.L. Jenkovszky, F. Paccanoni, E. Predazzi, Nucl. Phys. 25B, (Proc. Suppl.) (1992) 80.

17. M. Bertini et al., Phenomenological analysis of the small-x proton structure function in Strong Interaction at Long Distances (Hadronic Press), (1995) 181.

18. P. Desgrolard, L. Jenkovszky, F. Paccanoni, Eur. Phys. J. C7, (1999) 263.

19. P. Desgrolard, A. Lengyel, E. Martynov, Eur. Phys. J. C7, (1999) 655.

20. R. Fiore et al., Phys. Rev. D63, (2001) 056010.

21. H1 collaboration, T. Ahmed et al., Nucl. Phys. B439, (1995) 471.

H1 collaboration, S. Aid et al., Z. Phys. C69, (1995) 27.

H1 collaboration, S. Aid et al., Nucl. Phys. B470, (1996) 3. H1 collaboration, C. Adloff et al., Nucl. Phys. B497, (1997) 3.

H1 collaboration, C. Adloff et al., Eur. Phys. J. C13, (2000) 609.

H1 collaboration, C. Adloff et al., DESY-00-181, hepex/0012053(2000).
H1 collaboration, C. Adloff et al., DESY-01-104, hepex/0108035(2001).

ZEUS collaboration, M. Derrick et al., Phys. Lett. B293, (1992) 465; Z. Phys. C63, (1994) 391.

H1 collaboration, S. Aid et al., Z. Phys. C69, (1995) 27. ZEUS collaboration, M. Derrick et al., Zeit. Phys. C72, (1996) 399.

ZEUS collaboration, J. Breitweg et al., Phys. Lett. B407, (1997) 432.

ZEUS collaboration, J. Breitweg et al., Eur. Phys. J. C7, (1999) 609.

ZEUS collaboration, J. Breitweg et al., Nucl. Phys. B487, (2000) 53.

ZEUS collaboration, S. Chekanov et al., DESY 01-064(2001), hep-ex/0105090.

NMC collaboration, P. Amaudruz et al., Phys. Lett. B259, (1992) 159.

NMC collaboration, M. Arneodo et al., Nucl. Phys. B483, (1997) 3.

BCDMS collaboration, A.C. Benevenuti et al., Phys. Lett. B223, (1989) 485.

E665 collaboration, M.R. Adams et al., Phys. Rev. D54, (1996) 3006. 

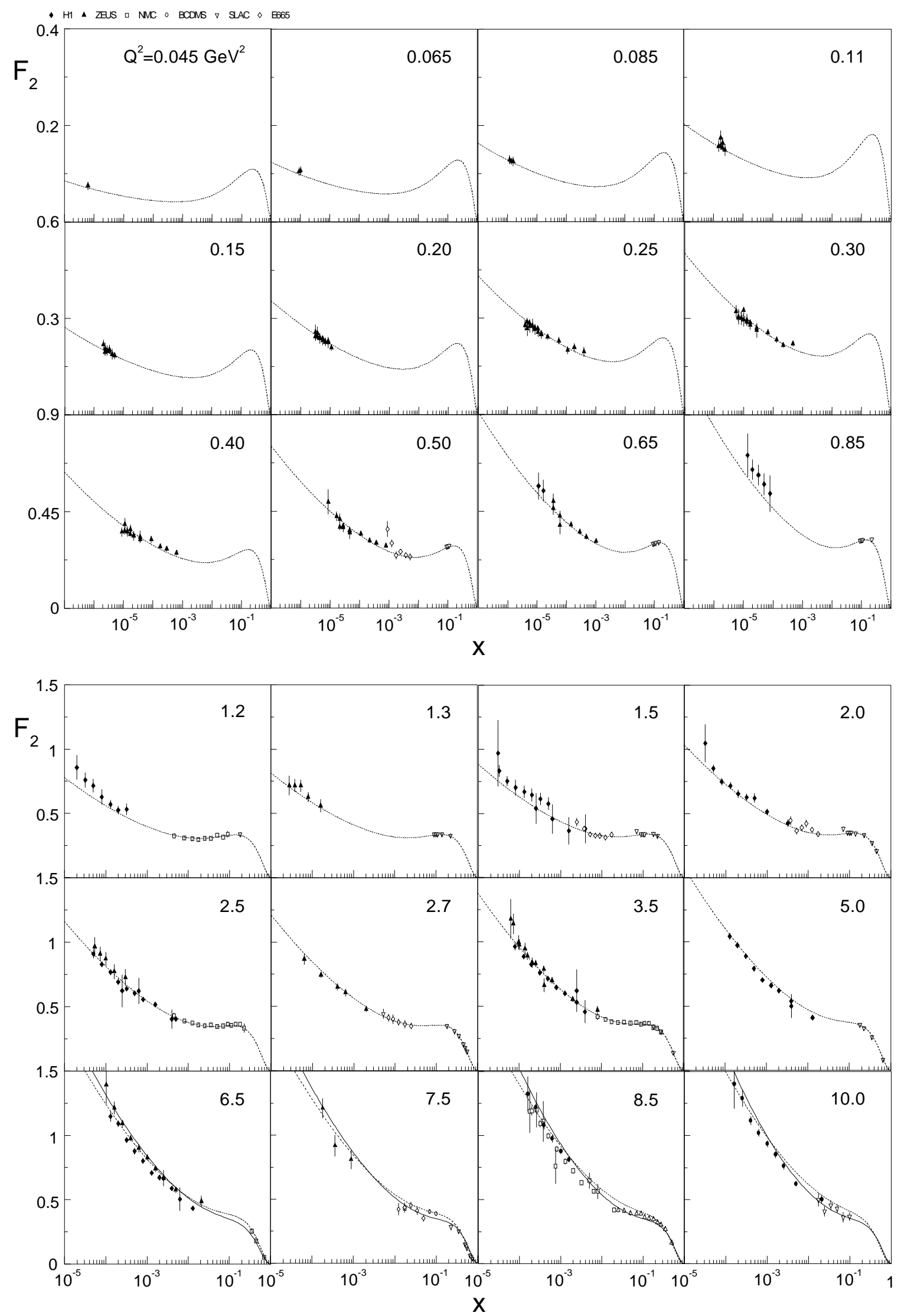

Fig. 2. Fit of $F_{2}$ for the smallest available values of $Q^{2}$ up to $5 \mathrm{GeV}^{2}$ and its extrapolation (dashed curve). The solid curve displays the result of evolution. 


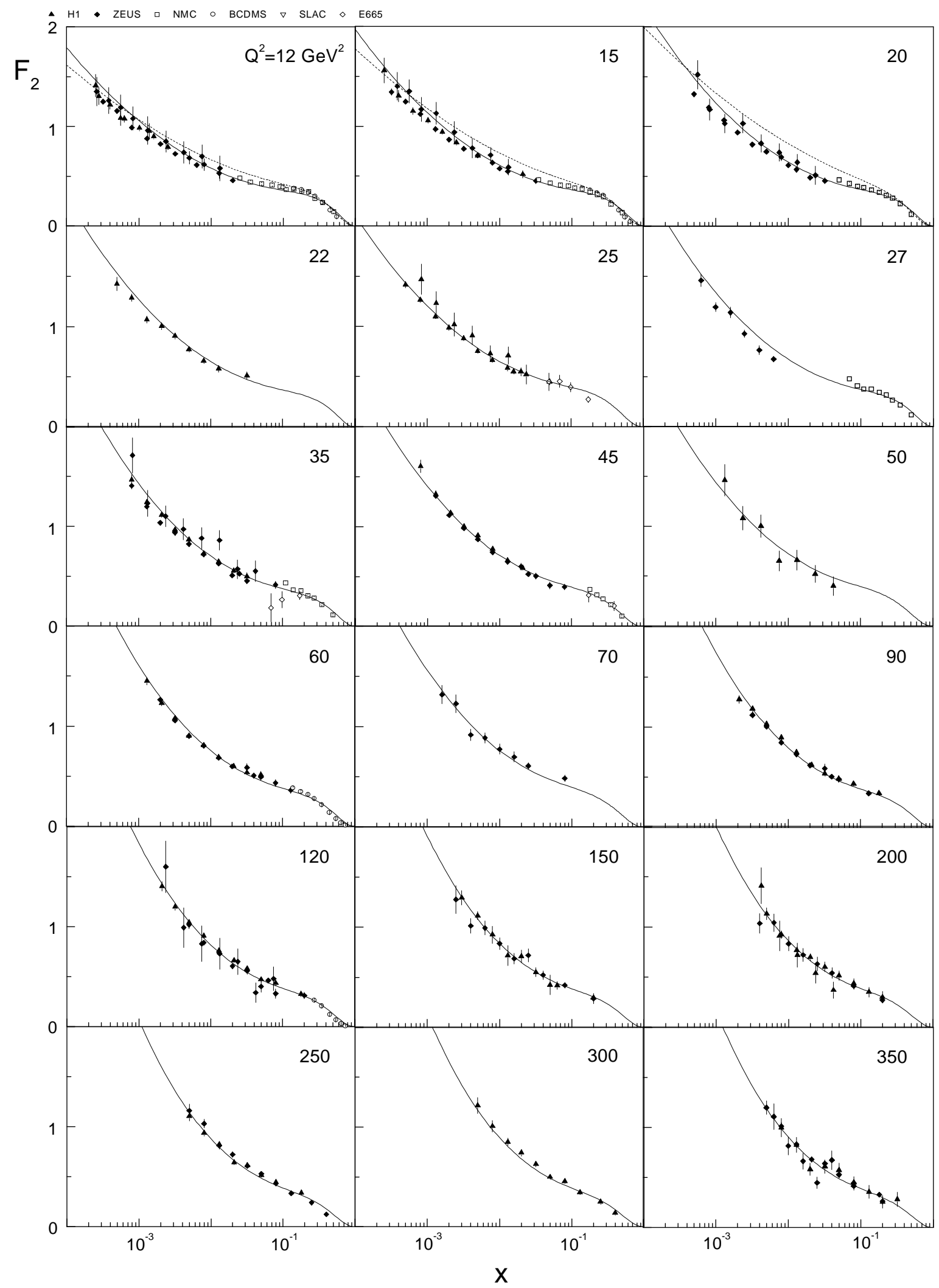

Fig. 3. The same as in Fig. 2. 
L. Csernai et al.: From Regge Behavior to DGLAP Evolution

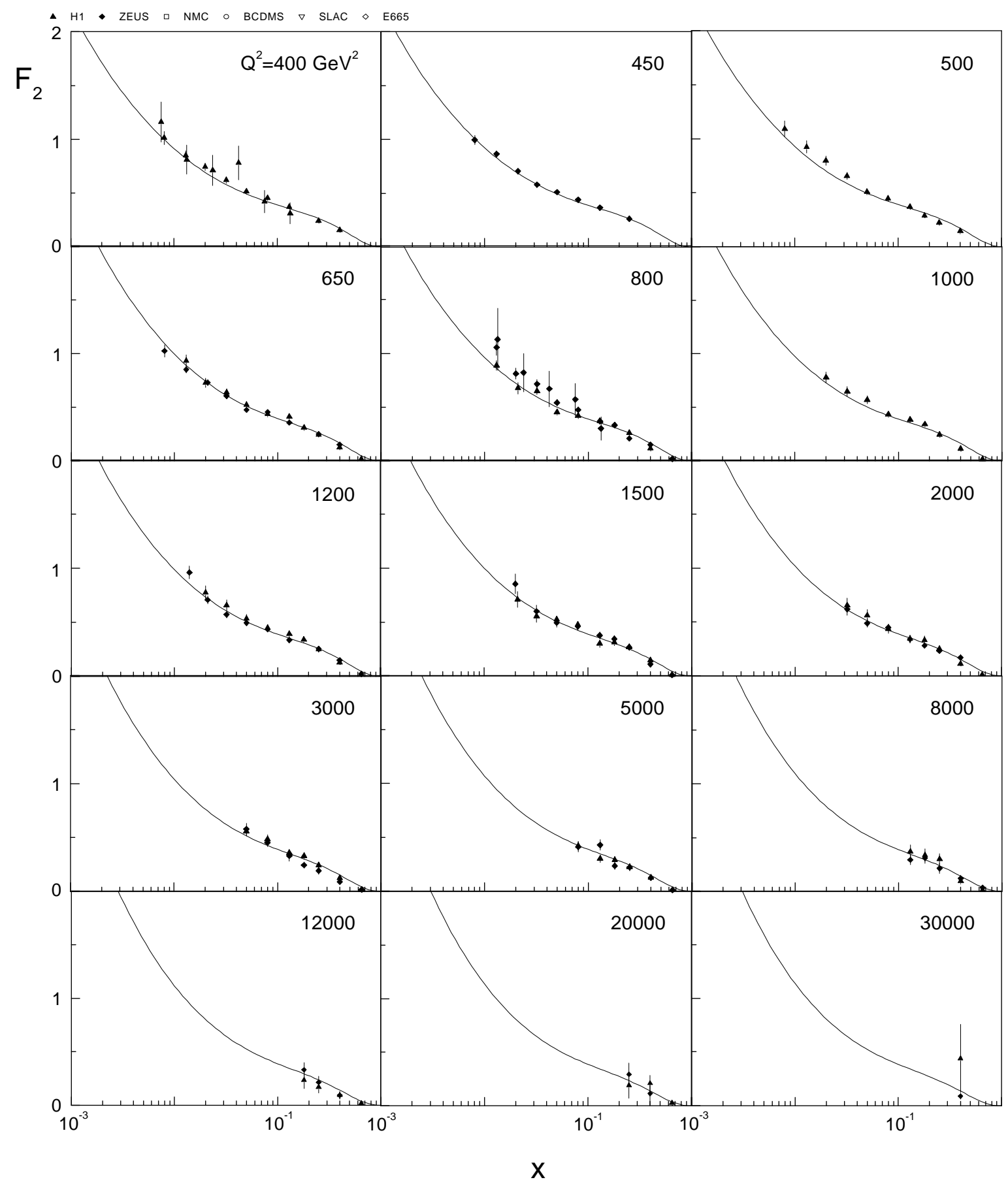

Fig. 4. Results for the evolution of $F_{2}$. 